\title{
DỨBin
}

Technological University Dublin ARROW@TU Dublin

\section{Assessing the time synchronisation of EEG systems}

\author{
Yongxiang Wang \\ Technological University Dublin \\ Charles Markham \\ National University of Ireland, Maynooth, charles.markham@mue.ie \\ Catherine Deegan \\ Technological University Dublin, catherine.deegan@tudublin.ie
}

Follow this and additional works at: https://arrow.tudublin.ie/itbengcon

Part of the Systems and Communications Commons

\section{Recommended Citation}

Y. Wang, C. Markham and C. Deegan, "Assessing the time synchronisation of EEG systems," 2019 30th Irish Signals and Systems Conference (ISSC), 2019, pp. 1-6, doi: 10.1109/ISSC.2019.8904947.

This Conference Paper is brought to you for free and open access by the Department of Engineering at ARROW@TU Dublin. It has been accepted for inclusion in Conference Papers by an authorized administrator of ARROW@TU Dublin. For more information, please contact arrow.admin@tudublin.ie, aisling.coyne@tudublin.ie, gerard.connolly@tudublin.ie. Funder: Technological University Dublin 


\section{Assessing the time synchronisation of EEG systems}

\author{
Yongxiang Wang \\ Department of Engineering \\ Technological University Dublin \\ Blanchardstown Campus \\ Dublin 15, Ireland \\ yongxiang.wang@mydit.ie
}

\author{
Charles Markham \\ Department of Computer Science \\ National University of Ireland \\ Maynooth, Ireland \\ charles.markham@mu.ie
}

\author{
Catherine Deegan \\ Technological University Dublin \\ Blanchardstown Campus \\ Dublin 15, Ireland \\ catherine.deegan@itb.ie
}

\begin{abstract}
This study compared the synchronisation of a medical grade Electroencephalography (EEG) system, the g.Tec, and a consumer grade EEG system, the Emotiv. Data was collected from both systems using the lab streaming layer (LSL). Both EEG systems recorded an electric signal from the surface of a customised gel phantom. The electric signal was generated using a solar cell which was illuminated by a monitor presenting a sequence of black and white images. Test results show that the g.Tec had a mean delay of $\mathbf{5 1 . 2 2} \mathbf{~ m s}$ from the stimulus onset and the Emotiv had a mean delay of $\mathbf{1 6 2 . 6 9} \mathrm{ms}$ from the stimulus onset. The result should be taken into account with future ERP studies which will use either the EEG system and the lab streaming layer. The design of this experiment provides a smart way to evaluate the temporal accuracy of other EEG systems.
\end{abstract}

Keywords-EEG, phantom, synchronisation, lab streaming layer, g.Tec, Emotiv

\section{INTRODUCTION}

In the fields of brain science, cognitive psychology and medical diagnosis, Electroencephalography (EEG) is usually involved and plays an important role. EEG is a noninvasive measurement, it considered as one of the safest ways to capture and monitor human brain activity. EEG uses electrodes, which placed along the scalp, measure the fluctuations in potential generated by the current flowing within the neurons of the brain [1]. EEG is one of the few techniques that provide millisecond range temporal resolution of brain activity. The use of EEG in conjunction with timed stimulus and other diagnostic techniques requires accurate timing and verified synchronisation of the data recorded from each sensor. This paper describes a method using a gel phantom and a photo sensitive electrode circuit that achieves this goal.

The medical grade EEG systems such as g.tec [2], BioSemi [3], NeuroScan [4] have been used in research and medical clinics for many years due to their high quality and reliability. In recent years, the popularity of inexpensive wireless EEG devices have been brought to researchers' attention. These wireless devices no longer need to restrict the EEG experiments to a specialist (shielded) laboratory, in addition they simplify the application of the electrodes. The wireless EEG devices are typically very easy to set up, using dry electrodes attached with cotton pads that are soaked in saline solution. These need only minor adjustments after headset is placed on the subject's head to achieve good EEG signal acquisition. The quality of the research findings for the wireless EEG systems have been comparable to the traditional EEG systems [5]. The most popular consumer grade EEG devices are the NeuroSky MindWave ${ }^{\mathrm{TM}}$ [6], the NeuroFocus Mynd $^{\mathrm{TM}}$, the Muse ${ }^{\mathrm{TM}}$ [7] and the Emotiv EPOC ${ }^{\mathrm{TM}}$ [8]. In the literature, there are an increasing number of research studies completed using the wireless systems, but there has not been a research study that have looked at the temporal accuracy of

This work is funded by the Technological University Dublin the Fiosraigh scholarship programme. these systems. Looking at all wireless EEG systems, the Emotiv has been used the most in research studies [9]. In this study, we will compare a medical grade EEG system, the g.Tec with the Emotiv EPOC+ EEG system.

Most research studies to date, that evaluated an EEG system, have used EEG spectrum analysis or the quality of the Event-related Potential (ERP) signal as a means of analysis. Measuring the EEG spectrum reflects the distribution of the signal power over frequency. This method divides the EEG signal into bands of frequency, such as the alpha band $(8 \mathrm{~Hz}-$ $13 \mathrm{~Hz})$, the beta band $(14 \mathrm{~Hz}-30 \mathrm{~Hz})$ etc. The brain wave patterns derived from each frequency bands can help doctors and scientists to identify a certain disease, for example, a spike or a sharp wave may represent a seizure or epilepsy [10]. For the ERP measurement, it is used to investigate brain activity synchronised to a time-locked stimulus or physical activity in the human body. The research studies from Campbell et al. [11], Tong et al. [12], Debener et al. [13] have been using the detection of the P300 component to detect a specific task using the Emotiv system. The P300 component is a positive change in potential. In the literature, it is most often elicited using the oddball paradigm [14], where the subject detects an infrequent 'target' stimulus [15, 16]. Duvinage et al. [17, 18], Badcock et al. [5, 19] and Lissa et al. [20] carried out comparison experiments between a medical grade EEG system and an Emotiv system. Their studies generated a P300 ERP component from both EEG systems and made a direct comparison of potential difference and peak latency. Duvinage et al. reported the P300 classification rate under sitting and walking conditions between ANT system and Emotiv system were $85.7 \% / 81 \%$ and $78.5 \% / 74.5 \%$ using the $\mathrm{k}$-fold validation in a visual stimulus paradigm. The signal-tonoise ratio is significantly worse in the Emotiv system. Badcock et al. compared P300 generated from auditory stimulus between Neuroscan system and Emotiv using frontal site electrodes. The P300 amplitude was $3.61 \mu \mathrm{V}$ vs $4.32 \mu \mathrm{V}$, the peak latency was $3.48 \mathrm{~ms}$ vs. $3.56 \mathrm{~ms}$. The results showed no statistically significant difference. Lissa et al. used Neuroscan system and Emotiv system to measure the N170 component using visual face stimuli. The experiment successfully replicated two key effects reliably found in ERP studies on face processing. In our study, we focused on the latency introduced by EEG systems prior to the pre-processing stage.

Although measuring the P300 is becoming a classic technique to evaluate an EEG system, there still exists the need to verify acquisition timings. The research studies, which measured the peak latency of the P300 component, do not investigate how much time the EEG signal spent in transmission, recording and pre-processing for the EEG systems. Almost every EEG system has its own recording application that is specially developed. A recording application used for an EEG device is very likely incompatible for a difference device. For this study, choosing a recording 
application that allows comparison of the two EEG systems is necessary.

The two most popular open-source applications to record EEG, to our knowledge are the OpenViBE [21] and the Lab Streaming Layer (LSL) [22]. In this study, we used LSL to record EEG signals. LSL records data as a streaming type. This application was chosen because our future experiments will synchronise an EEG device, an eye tracking device (ET) and a galvanic skin response device (GSR). The LSL repository has several ET and GSR modules that demonstrated its capability of synchronising and recording ET and GSR data together [22].

In order to validate timings, experimental conditions must be created such that the human subject is not used in the experiment. The introduction of a human causes subject-to subject variability [23], as well as measurement variability [24], which would adversely affect the timing measurements of any delays in the hardware. The EEG measured from electrodes are the summary of potential variations generated from multiple locations. A minor change by visual, auditory or sensory stimulus will make the EEG signal look different. Events such as random eye blinks, heartbeats, muscle movement can create significant artefacts in the EEG signal.

In this study, we fabricated a gel phantom to simulate the human brain, and applied a measurable potential difference which was synchronised with the stimulus appearing on the monitor.

\section{Methodology}

\section{A. Phantom fabrication and property}

Our approach to measuring the latency caused by the data transfer from the EEG devices to the recording application on the desktop machine was organised in the following manner. First we simulated the human brain by fabricating a gel phantom head. Using a ratio of $1000 \mathrm{~g}$ of water to $2 \mathrm{~g} \mathrm{NaCl}$ salt (sodium chloride) to 108 grams beef gelatine $(9$ sachets Dr Oetker), a phantom material solution was created. The solution was heated and stirred in a saucepan until the gelatine was dissolved, boiling was avoided. The solution was set in a plastic serving bowl (12 cm diameter) lined with clingfilm. Electrodes consisting of $8 \mathrm{~mm}$ brass washers on which were soldered leads were positioned in the gel. The leads were made by winding insulated multistrand wire around a former to create a 5 turn spring, so as to reduce the chance of the electrode being pulled out of the gel. The material was kept in the fridge to cool and harden at 4 degree Celsius overnight ( 8 hours approximately), producing a final gel phantom model as shown in Fig. 1. The electrodes were positioned in a straight line, $3 \mathrm{~cm}$ distance between each other.

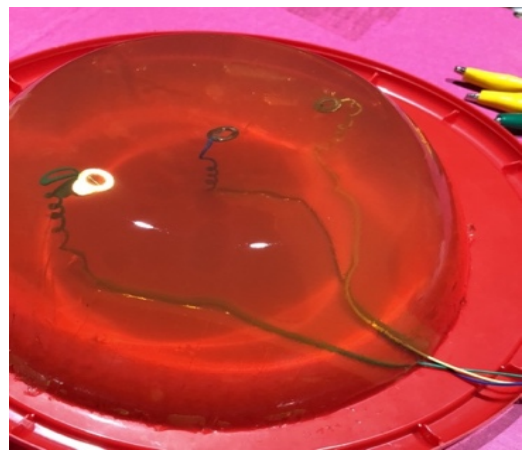

Fig. 1 The final gel phantom model. Three electrodes embedded inside the phantom, left electrode soldered to green wire, middle electrode soldered to yellow wire, right electrode soldered to blue wire.

\section{B. Experiment design}

The experiment used a solar cell to generate a simulated signal to send into the phantom, see Fig. 2. The solar cell provided a means of generating the signal to compare the timing of the event marker with the EEG signal. In hardware triggered ERP studies, it's typical to use something like a photodiode to detect changes (e.g. switching black and white images) in the corner of a screen and output these changes as markers directly into the EEG [5]. The approach used in this work allowed the same protocol to be used as in the experiment on human subjects. The only change required was to replace the target images with white images and non-target images with black. The positive terminal of the solar cell was connected to the central electrode of the phantom. The negative terminal was connected to the electrodes $3 \mathrm{~cm}$ on the right side of the central electrode shown in Fig. 1. The other electrode at the left side of the central electrode was not used. A $100 \mathrm{ohm}$ resistor connected in parallel with the solar cell, was used to improve the response time of the solar cell and overcome any delay caused by the internal capacitance of the solar cell (and phantom). The solar cell was then attached to the bottom right corner of a 19-inch computer monitor (Medion AKOYA X54000 MD 20165). The monitor was set to be the highest refresh rate, $75 \mathrm{~Hz}$. The stimulus used a total of 505 black images ( $800 \times 600$ pixels) as non-target stimuli and a total of 127 black images with white rectangular $(150 \mathrm{x}$ 126 pixels) at the bottom right corner as target stimuli, shown in Fig. 3. All images were randomly shuffled and presented on the monitor with a time interval of one second between images. When black image was displayed on screen, there was no potential difference measured by the solar cell, when the black image containing a white image region was displayed on screen, there was an approximate $13.48 \mathrm{mV}$ potential generated by the solar cell circuit.

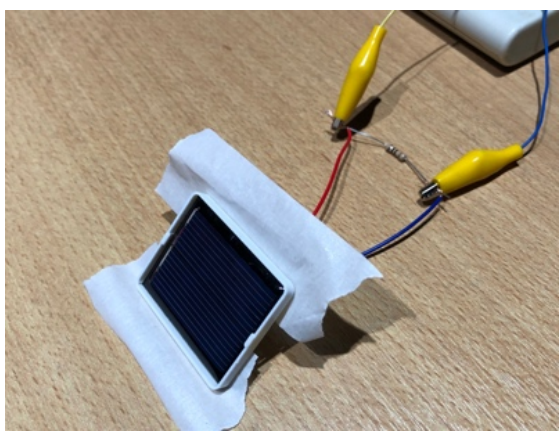

Fig. 2 A solar cell unit obtained from a solar cell toy.
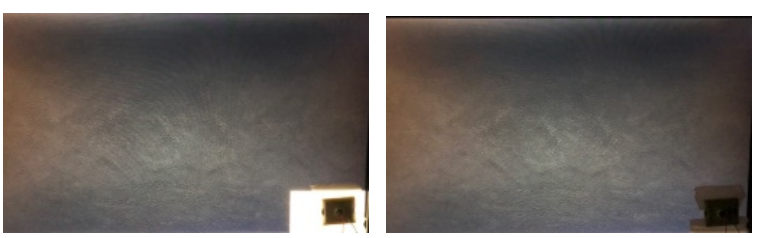

Fig. 3 Photographs of the monitor. The left figure is the target stimulus image black, the right figure is the non-target stimulus image black with a white rectangular region at bottom right corner.

All image stimuli were displayed using the software Psychopy (version 1.85.2) [25]. The software generated software triggers which marked the target and non-target 
image. These triggers were sent to EEG acquisition program directly that run on the same computer as an LSL stream.

\section{Equipment}

The two EEG systems that were evaluated in the experiment were a medical grade g.Tec g.USBamp system and a consumer grade Emotiv EPOC + system (2016 later edition) as shown in Fig. 4. The g.Tec g.USBamp system uses a standard USB 2.0 interface, which makes the amplifier straightforward to connect to the USB socket on PC/notebook and can immediately be used for data recording. The system has 16 simultaneously sampled biosignal channels each with 24 bits resolution. The sampling rate can adjust from $64 \mathrm{~Hz}$ to $38.400 \mathrm{~Hz}$. In this experiment, the electrode impedance was measured and maintained under $20 \mathrm{k} \Omega$ for each channel by using conductive gel. Signals were checked using the g.Tec Matlab Simulink impedance checker.

The Emotiv EPOC+ headset comes with 14 channels and uses CMS/DRL (common mode sense/driven right leg) references at P3/P4 locations. The electrodes are held with a plastic arm that holds a small cap with saline soaked felt pad inside. The electrode locations are based on the international 10-20 system. The device uses a Bluetooth transmitter and has an inbuilt battery with a life of about 12 hours. The signal is internally digitized at $2048 \mathrm{~Hz}$ (16-bit) and subsequently low pass filtered $(43 \mathrm{~Hz})$ and downsampled to either $128 \mathrm{~Hz}$ or $256 \mathrm{~Hz}$.
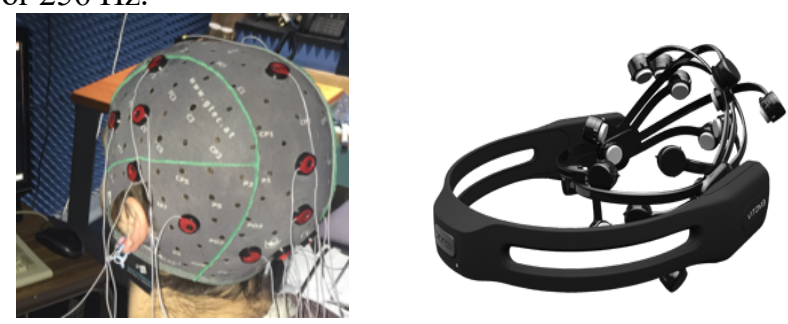

Fig. 4 The two EEG systems studied. The left figure is the medical grade EEG system, g.Tec g.USBamp. The right figure is the consumer grade EEG system, Emotiv EPOC+ [8].

\section{EEG recording}

In EEG studies, especially for the ERP measurement, the timing accuracy is critical. We used an open source software called lab streaming layer (LSL) [22] to synchronise the EEG data recordings and the event onset markers. The LSL system is specified to measure time series in research experiments. LSL handles both the networking, time-synchronization, (near-) real-time access as well as optionally the centralized collection, viewing and disk recording of the data [22]. The LSL supports both the g.tec system and the Emotiv system.

The experiment took place in a radio frequency (RF) shielded room $(>100 \mathrm{~dB} 1 \mathrm{GHz}$ and $100 \mathrm{GHz})$. When recording the simulated input signal, the g.Tec system amplifier and the Emotiv Bluetooth dongle were connected to a desktop computer running the LabRecorder, a default recording program that comes with LSL. The experimental runs for each device were implemented one after the other. For the g.Tec system we recorded the simulated signal at 256 $\mathrm{Hz}$ sampling rate and used the reference electrode, ground electrode at the position shown in Fig. 5. We chose the reference and ground electrode location for the g.Tec was consistent with the reference sensors of Emotiv EPOC+. The electrode that was used for measuring the simulated input signal was T7. By cutting out $2 \mathrm{~cm}$ wide pieces of gel material from both sides of the phantom as shown in Fig. 5, we could fit the Emotiv EPOC+ headset firmly on the phantom. The Emotiv system recorded the simulated signal at $128 \mathrm{~Hz}$.

\section{E. EEG processing}

The data recorded from the g.Tec g.USBamp and the Emotiv systems were processed offline using EEGLAB (v 14.1.1) [23]. The continuous g.Tec data was downsampled from $256 \mathrm{~Hz}$ to $128 \mathrm{~Hz}$, in order to match the sampling rate of the Emotiv system. The data from the g.Tec and Emotiv were processed using finite impulse response (FIR) filter bandpass filtered at $0.16-30 \mathrm{~Hz}$ to remove DC and high frequency noise. Data from both systems was then extracted for epochs from $-200 \mathrm{~ms}$ to $1200 \mathrm{~ms}$ with respect to stimulus onset (0 ms).

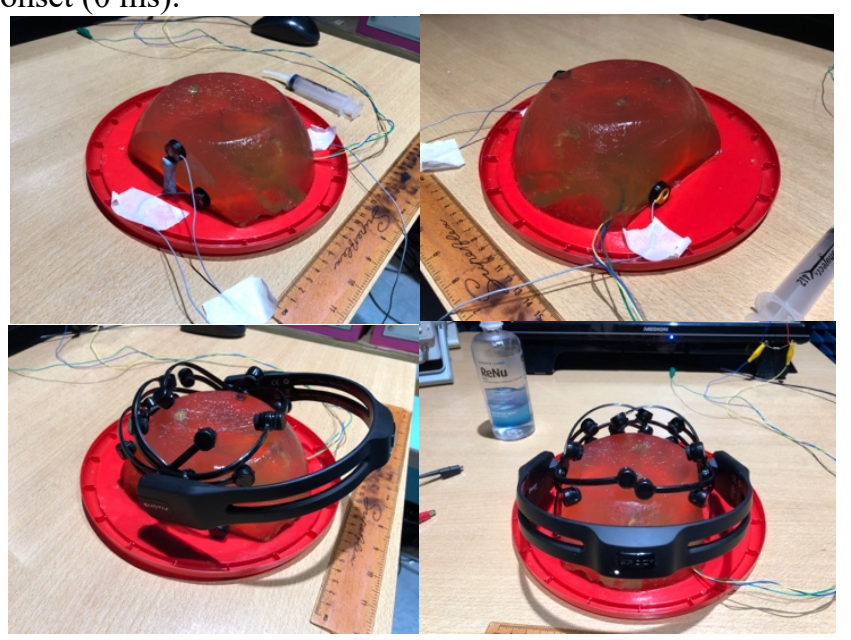

Fig. 5 Illustration of electrode locations used in the experiment. The top two figures are the EEG electrodes set up for the g. Tec system. The bottom two figures are the EEG headset set up for the Emotiv system. The reference location is the bottom electrode shown in top left figure, the ground electrode location is shown in the top right figure.

\section{RESULTS}

\section{A. Property of the electric circuit}

We first measured the rise time of the circuit used to produce a step signal following the stimulus image onset. An oscilloscope was used to measure the signal across the two solar cell wires, in parallel with the $100 \mathrm{ohms}$ resistor. The oscilloscope captured the response shown in Fig. 6. The measurement showed the distance between the rising edge and the falling edge is 1 second duration, which matched the image presentation frequency. The rising edge of the signal showed that the time spent for the solar cell to reach its saturated charging state was approximately 38 microseconds.

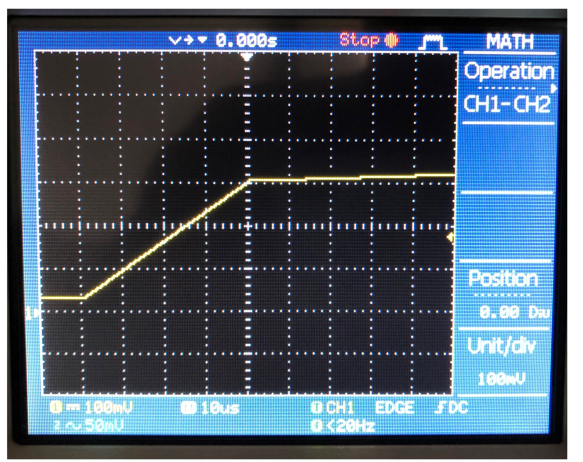

Fig. 6 Oscilloscope measurement of voltage generated by the solar cell excited by target image. The raising edge is zoomed in to measure the time spend for solar cell reach to the peak voltage $(\mathrm{x}=10 \mu \mathrm{s} / \mathrm{div}, \mathrm{y}=100 \mathrm{~ms} / \mathrm{div})$. 


\section{B. Temporal analysis of two EEG systems}

All data epochs were averaged from all trials recorded by each EEG system. The correspondent target stimulus epochs and non-target stimulus epochs were averaged respectively.

Fig. 7 presents the comparison of the simulated input signals recorded from both systems. The time zeros in each sub figures were the actual stimulus onset time for either target stimuli (black image with bottom right corner white image) or non-target stimuli (single black image). The image presentation sends a timestamp and image marker to the recording component for every stimulus.

Fig. 7 a. and b. were plotted using the signal trial ERP method in EEGLAB. The blue band is shown in Fig. 7 a. and $b$. indicated when the target images were onset and the red band indicated when the target images were removed. The negative going and positive going peaks were due to $\mathrm{AC}$
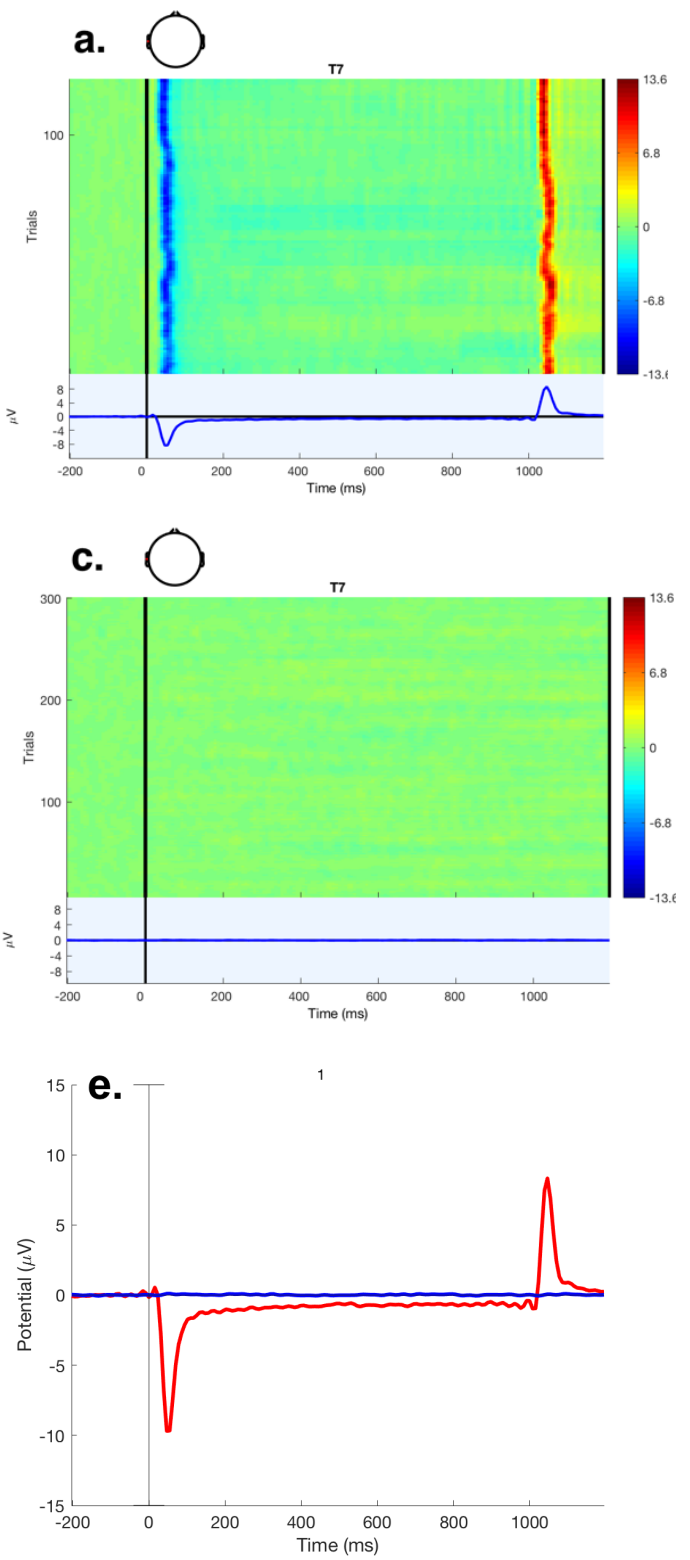

coupling. This means that the electrode potential (signal) is effectively the derivative of the signal produced across the solar cell. A consistent blue and red band represented the input signal generated by the target stimuli was consistently captured by both EEG systems. A significant delay can be observed as the gap between blue band and time zero in Fig. 7 b. This is wider than the gap in Fig. 7 a. Therefore, we know the time spent by the Emotiv system to record the simulated input signal was longer than the g.Tec system. Another observation from Fig. $7 \mathrm{a}$ and $\mathrm{b}$ shows that both the blue band and red band in the Emotiv system show more variation than the g.Tec system.

We also plotted the single trial ERP image for non-target image stimulus using same scales for both system as shown in Fig. $7 \mathrm{c}$. and d. The Fig. $7 \mathrm{c}$. and d. are in contrast with the $\mathrm{a}$ and $\mathrm{b}$ which has no significant signals were captured.
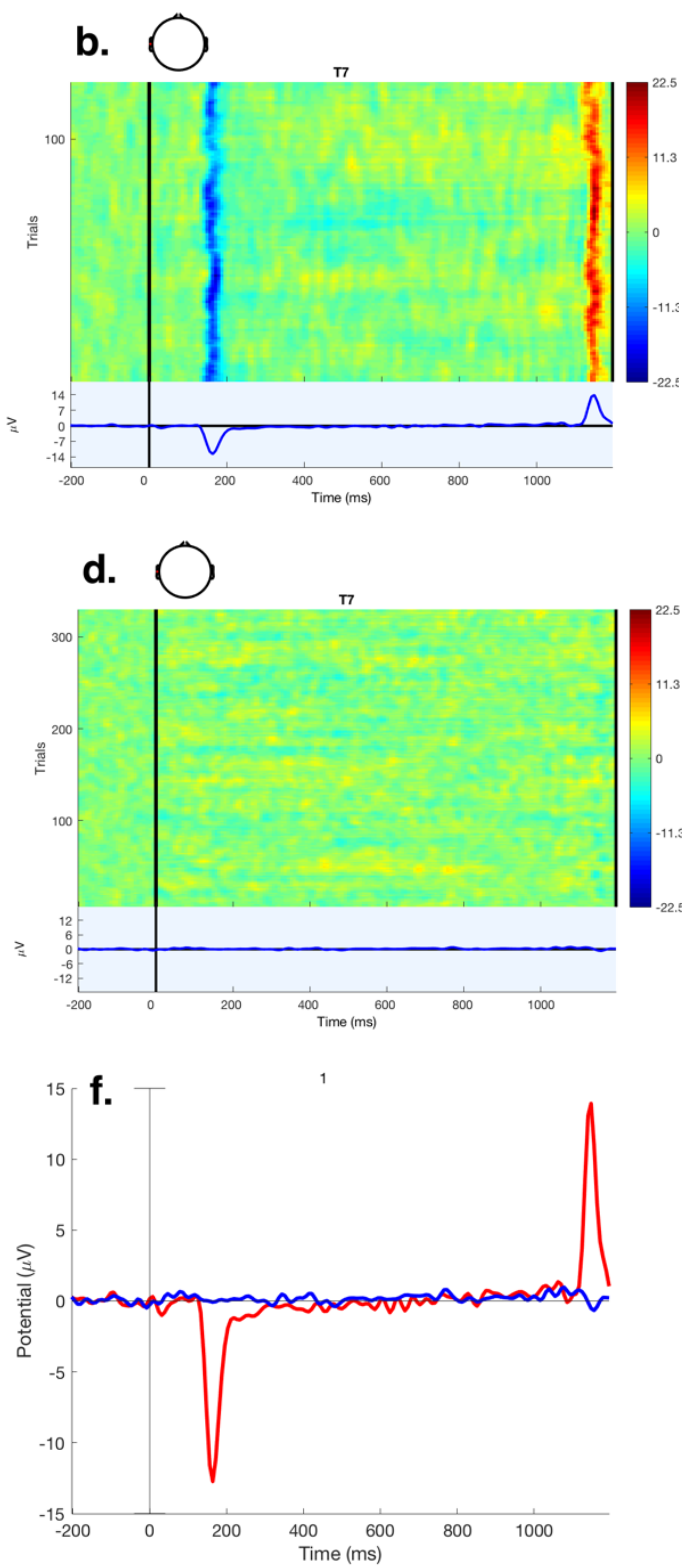

Fig. 7 The sub-figures of left column were recorded from the g.Tec system. The sub-figures of right column are recorded from the Emotiv system. (a.) and (b.) are single trial plots for target stimuli, 127 in total. (c.) and (d.) are the single trial plot for non-target stimuli, 505 in total. (e.) and (f.) are averaged plots for target stimuli (red) vs. non-target stimuli (blue) 
Fig. 7 e. and f. were plotted using the averaged ERP method in EEGLAB. Both plots compared the averaged target versus the averaged non-target image stimulus. In Fig. $7 \mathrm{e}$. and f., we can obtain some information. The delay from time zero to the averaged negative peak amplitude is approximately $51.22 \pm 0.89 \mathrm{~ms}(\mathrm{SD}=9.27)$ for the g.Tec system and $162.69 \pm 1.10 \mathrm{~ms}(\mathrm{SD}=11.40)$ for the Emotiv system. The time lag between the two systems was about $111.47 \mathrm{~ms}$. We chose the first sample point which initiated the negative transition as the time of image onset. The g.Tec system initiated recording at $23.44 \mathrm{~ms}$. The Emotiv system initiated recording at $125.00 \mathrm{~ms}$. The delay from the g.Tec system is small, and in some cases would not need to be compensated for in some future ERP studies, such as the P300 ERP studies. The Emotiv system has a much longer delay, this must be considered in future ERP studies, since a $100 \mathrm{~ms}$ delay difference can lead to incorrect identification of ERP components [16]. We observed the averaged peak amplitude for the g.Tec system is $-13.45 \pm 0.07 \mu \mathrm{V}$ ( $\mathrm{SD}=$ 0.77 ) and the averaged peak amplitude for the Emotiv system is $-22.56 \pm 0.50 \mu \mathrm{V}(\mathrm{SD}=5.25)$. Although the Emotiv signal is bigger than the $\mathrm{g}$.Tec, this is not a comment on the quality of the signal. We also observed during the transition period between the negative peak and the positive peak, the averaged signals recorded by the $\mathrm{g}$. Tec system has less variation than the Emotiv system.

\section{Signal to noise ratio}

To measure the quality of the recorded signals in both EEG systems, we measured the signal to noise ratio (SNR) for both averaged waveforms (Fig. 7 e. and f.).

$$
\begin{aligned}
& V_{r m s}=\sqrt{\frac{\sum\left(V_{n}^{2}\right)}{n}} \\
& S N R=\left(\frac{V_{\text {signal }}}{V_{\text {noise }}}\right)^{2} \\
& S N R_{d B}=10 \log _{10} S N R
\end{aligned}
$$

We used both negative and positive peak signals in both averaged target waveforms to increase the number of sample points to calculate the $\mathrm{V}_{\text {signal, }}$, in order to improve the accuracy of the SNR. We selected 22 sample points (31 to 41 and 155 to 165$)$ as signal and 41 sample points (100 to 140$)$ as noise between the two averaged target waveforms (Fig. 7 e. and f.). Using the same number of sample points to make sure the comparison is consistent. A DC offset was removed using the mean over the sample. The RMS value was then calculated on the data. The SNR of the averaged target waveform for the g.Tec system is $39.93 \mathrm{~dB}$ and the SNR of the averaged target waveform for the Emotiv system is $26.34 \mathrm{~dB}$. In the shielded room the g.Tec is performing better. However further studies would be required to compare the performance of the two systems when used in environments where interference is present, as they use different approaches to handle common mode signals.

\section{DISCUSSIONS}

The aim of this paper is to compare and validate the time accuracy between a medical grade EEG system and a consumer grade EEG system each acquiring data using LSL. The result of the experiment showed the consumer grade EEG system has a longer time lag when recording a signal with the LSL compare to the medical grade EEG system. In our previous study, we demonstrated the capability of the Emotiv system to measure the P300 ERP waveform compared to the g.Tec system, which the Emotiv system can produce useful ERP waveforms, but there was a $125 \mathrm{~ms} \mathrm{lag}$ compared to the g.Tec system [26]. The result of this paper in line with our previous study [26].

In previous work systems were compared using data recorded from human participants. The method developed in this paper uses a novel photo-sensitive phantom to uniform generate signals which simplifies the analysis of the temporal response of EEG equipment. The proposed method can be easily applied to the evaluation of other EEG and physiological measurement systems.

The input signal applied to the phantom was measured with a digital storage oscilloscope. A square wave transient was recorded each time the stimulus was present, as shown in Fig. 8. The origin of the negative peak and a positive peaks corresponding to the target stimulus image onset and conclusion. Its appearance suggest that capacitive coupling between the inner electrodes and the EEG electrodes dominates. We simulated the phantom and solar cell using a simple RC circuit as shown in Fig. 9. The output of this circuit can produce a similar response as the phantom as shown in Fig. 10. We believe that a series RC circuit provides a good model of the response.

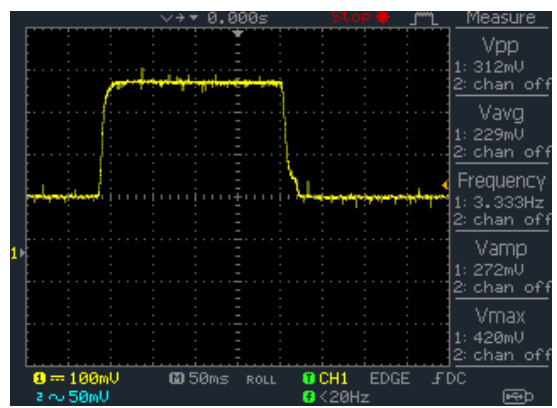

Fig. 8 Oscilloscope measured input signal generated by solar cell.

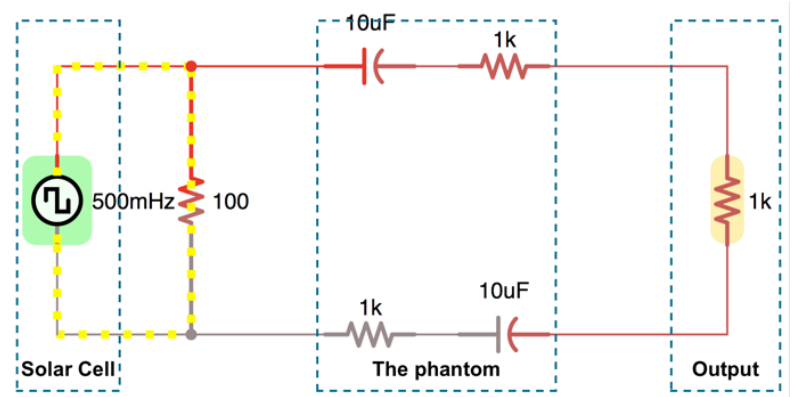

Fig. 9 Simulated electronic circuit for the setup of solar cell and phantom using the iCircuit App [27].

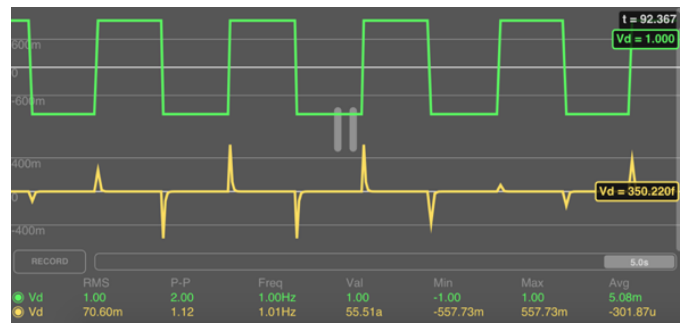

Fig. 10 The simulation result from Fig. 9. The result demonstrated a similar response as using the gel phantom. The green signal is the input signal (square wave), the yellow signal is the output signal. The output signal has been capacitively coupled. 
The averaged waveform of target image stimuli recorded from the Emotiv system has a higher potential then the g.Tec system. The g.Tec had an improved signal to noise. However this improved signal was only demonstrated in an environment with very low levels of electromagnetic interference (EM) interference. The circuitry used by each system to handle noisy environments is different and this comparison would require further measurement.

\section{LIMITATIONS}

In this paper, we simply set the computer monitor to its highest refresh rate $(75 \mathrm{~Hz})$. We did not investigate the delay between image onset time and computer screen frame rate (the effect of double buffering). Using a $75 \mathrm{~Hz}$ monitor, the mean latency between request a re-drawing and image appearing is approximate $6.71 \mathrm{~ms}(\mathrm{SD}=3.87)$. This latency is much small than the latencies measured in an ERP study. It always after the onset event. We are confident that there's a greater lag time for the Emotiv vs g.tec.

\section{CONCLUSION}

This project used a gel phantom stimulated by a photosensitive circuit to measure the synchronisation of both the g.Tec EEG and the Emotiv EEG recorded by LSL. The result from the experiment determined the delay of g.Tec with LSL is $51.22 \mathrm{~ms}$ and the delay of Emotiv with LSL is 162.52 $\mathrm{ms}$. The measured delay from both systems can help future ERP experiments, which use LSL.

\section{Acknowledgment}

The authors would like to thank Dr. Tomas Ward for the loan of the g.Tec EEG system and Dr. David Malone for providing the RF shielded room at the Hamilton Institute at Maynooth University to do the study. In addition, this work was supported by funding from the Technological University Dublin Fiosraigh scholarship programme.

\section{REFERENCES}

[1] S. Sanei and J. A. Chambers, EEG Signal Processing (no. Book, Whole). Wiley, 2013.

[2] "G.Tec EEG system produce description. ," 2013. Available: https://gtecmedical.wordpress.com/2013/11/05/new-wirelessactive-eeg-system/. [Accessed: 21 May 2016]

[3] "BioSemi EEG active-two product description." Available: https://www.biosemi.com/products.htm. [Accessed: 15 Feb 2019]

[4] "NeuroScan EEG solutions and products." Available: https://compumedicsneuroscan.com/applications/eeg/. [Accessed: 30 Jan 2019]

[5] N. A. Badcock, P. Mousikou, Y. Mahajan, P. de Lissa, J. Thie, and G. McArthur, "Validation of the Emotiv EPOC® EEG gaming system for measuring research quality auditory ERPs," PeerJ, vol. 1, p. e38, 2013.

[6] "Neurosky - Mindwave brain sensing headband." Available: http://store.neurosky.com/pages/mindwave. [Accessed: 01 Feb 2018]

[7] "Muse - the brain sensing headband." Available: http://www.choosemuse.com. [Accessed: 05 Feb 2018]
[8] "Emotiv - Epoc bain sensing headband." Available: https://www.emotiv.com. [Accessed: 01 Feb 2018]

[9] R. Yannick, B. Hubert, A. Isabela, G. Alexandre, and F. Jocelyn, "Deep learning-based electroencephalography analysis: a systematic review," arXiv preprint arXiv:1901.05498, 2019.

[10] R. S. Fisher et al., "Epileptic seizures and epilepsy: definitions proposed by the International League Against Epilepsy (ILAE) and the International Bureau for Epilepsy (IBE)," Epilepsia, vol. 46, no. 4, pp. 470-472, 2005

[11] A. Campbell et al., "NeuroPhone: brain-mobile phone interface using a wireless EEG headset," 2010, pp. 3-8: ACM.

[12] T. Jijun, Z. Peng, X. Ran, and D. Lei, "The portable P300 dialing system based on tablet and Emotiv Epoc headset," 2015, pp. 566569: IEEE.

[13] S. Debener, F. Minow, R. Emkes, K. Gandras, and M. Vos, "How about taking a low - cost, small, and wireless EEG for a walk?," Psychophysiology, vol. 49, no. 11, pp. 1617-1621, 2012.

[14] J. Polich and C. Margala, "P300 and probability: comparison of oddball and single-stimulus paradigms," International Journal of Psychophysiology, vol. 25, no. 2, pp. 169-176, 1997.

[15] D. E. J. Linden, "The P300: where in the brain is it produced and what does it tell us?," The Neuroscientist, vol. 11, no. 6, pp. 563$576,2005$.

[16] S. J. Luck, An Introduction to the Event-Related Potential Technique (A Bradford book, no. Book, Whole). MIT Press, 2014.

[17] M. Duvinage, T. Castermans, M. Petieau, T. Hoellinger, G. Cheron, and T. Dutoit, "Performance of the Emotiv Epoc headset for P300based applications," Biomedical engineering online, vol. 12, no. 1, p. 56, 2013.

[18] M. Duvinage et al., "A P300-based quantitative comparison between the Emotiv Epoc headset and a medical EEG device," Biomedical Engineering, vol. 765, no. 1, pp. 2012-2764, 2012.

[19] N. A. Badcock et al., "Validation of the Emotiv EPOC EEG system for research quality auditory event-related potentials in children," PeerJ, vol. 3, p. e907, 2015.

[20] P. de Lissa, S. Sörensen, N. Badcock, J. Thie, and G. McArthur, "Measuring the face-sensitive N170 with a gaming EEG system: A validation study," Journal of Neuroscience Methods, vol. 253, pp. 47-54, 2015/09/30/ 2015.

[21] Y. Renard et al., "OpenViBE: an open-source software platform to design, test, and use brain-computer interfaces in real and virtual environments," Presence: teleoperators and virtual environments, vol. 19, no. 1, pp. 35-53, 2010.

[22] "Lab Streaming Layer software description." Available: https://github.com/sccn/labstreaminglayer. [Accessed: 20 Oct 2018]

[23] J. Intriligator and J. Polich, "On the relationship between EEG and ERP variability," International Journal of Psychophysiology, vol. 20, no. 1, pp. 59-74, 1995.

[24] E. Callaway and R. A. Halliday, "Evoked potential variability: Effects of age, amplitude and methods of measurement," Electroencephalography and clinical neurophysiology, vol. 34, no. 2, pp. 125-133, 1973.

[25] J. W. Peirce, "PsychoPy-psychophysics software in Python," Journal of neuroscience methods, vol. 162, no. 1-2, pp. 8-13, 2007.

[26] Y. Wang, Z. Wang, W. Clifford, C. Markham, T. E. Ward, and C. Deegan, "Validation of low-cost wireless EEG system for measuring event-related potentials," 2018, pp. 1-6: IEEE.

[27] "iCircuit application - electronic circuit simulator and designer." Available: http://icircuitapp.com. [Accessed: 30 Jan 2019] 